\title{
SIMULTANEOUS FITS IN ISIS ON THE EXAMPLE OF GRO J1008-57
}

\author{
M. Kühnel ${ }^{a, *}$, S. MÜller ${ }^{a}$, I. Kreykenbohm ${ }^{a}$, F.-W. Schwarm ${ }^{a}$, \\ C. Grossberger ${ }^{a}$, T. Dauser ${ }^{a}$, M. A. Nowak ${ }^{b}$, K. Pottschmidt ${ }^{d, c}$, \\ C. Ferrigno ${ }^{g}$, R. E. Rothichild ${ }^{e}$, D. Klochkov $^{f}$, R. $\operatorname{Staubert}^{f}$, \\ J. WiLMS ${ }^{a}$
}

${ }^{a}$ Remeis-Observatory \& ECAP, Universität Erlangen-Nürnberg, Sternwartstr. 7, 96049 Bamberg, Germany

${ }^{b}$ MIT Kavli Institute for Astrophysics, Cambridge, MA 02139, USA

${ }^{c}$ CRESST, Center for Space Science and Technology, UMBC, Baltimore, MD 21250, USA

d NASA Goddard Space Flight Center, Greenbelt, MD 20771, USA

e Center for Astronomy and Space Sciences, University of California, San Diego, La Jolla, CA 92093, USA

$f$ Institut für Astronomie und Astrophysik, Universität Tübingen, Sand 1, 72076 Tübingen, Germany

g ISDC Data Center for Astrophysics, Chemin d'Écogia 16, 1290 Versoix, Switzerland

* corresponding author: matthias.kuehnel@sternwarte.uni-erlangen.de

\begin{abstract}
Parallel computing and steadily increasing computation speed have led to a new tool for analyzing multiple datasets and datatypes: fitting several datasets simultaneously. With this technique, physically connected parameters of individual data can be treated as a single parameter by implementing this connection directly into the fit. We discuss the terminology, implementation, and possible issues of simultaneous fits based on the Interactive Spectral Interpretation System (ISIS) X-ray data analysis tool.While all data modeling tools in X-ray astronomy in principle allow data to be fitted individually from multiple data sets, the syntax used in these tools is not often well suited for this task.

Applying simultaneous fits to the transient X-ray binary GRO J1008-57, we find that the spectral shape is only dependent on X-ray flux. We determine time independent parameters, e.g., the folding energy $E_{\text {fold }}$, with unprecedented precision.
\end{abstract}

KEYwordS: Methods: data analysis - X-rays: binaries - (Stars:) pulsars: individual GRO J1008-57.

\section{Motivation}

Most data analysis in X-ray astronomy concentrate on describing single datasets or on characterizing samples with the results of fits of individual datasets. Once a good description of an example dataset is found, an analysis of comparable datasets follows. Finally, the results of all those individual analyses are compared and interpreted.

For example, a particular parameter is found to depend on other parameters. Instead of going back to the data analysis and fitting this dependency directly to enhance the precision of the parameter or to break degeneracies (feasible through reduced degrees of freedom), the dependency is then analyzed on its own. In another way, the former analysis is indeed repeated but with this parameter fixed according to the dependency that has been discovered. Furthermore, if parameters cannot be constrained well, these parameters are commonly fixed to a certain standard value.

We therefore cannot gain any physical information from this fixed parameter and, more importantly, systematical effects may arise. The reason for not adopting sophisticated ways is usually a lack of computation power. Implementing parameter correlations or de- pendencies would require all data to be analyzed at the same time. However, since computer power has increased and parallel computation using several computers is possible, the situation has now changed. In other words, fitting data simultaneously has become feasible even when large numbers of datasets (e.g., 50100 pointings at a single source) are to be considered.

In Section 2 we introduce an implementation of simultaneous data analysis into the Interactive Spectral Interpretation System (ISIS) [1, which has been designed to "facilitate the interpretation and analysis of high resolution X-ray spectra' 17 In Section 3 , we present ideas for possible applications of simultaneous data analysis and further demonstrate the power of this method on the example of the transient Xray binary GRO J1008-57 in Section 4 Finally, we discuss questions and issues, which arise by comparing advantages and disadvantages of simultaneous fits (Table 1).

\section{IMPLEMENTATION INTO ISIS}

ISIS 11 was developed to fit X-ray spectra, but it can also be used to analyze almost all kinds of data, due to its strong customization capability [2] compared

\footnotetext{
${ }^{1}$ http://space.mit.edu/CXC/ISIS/ [2015-04-01]
} 


\section{Advantages}

- Fixed parameters can be determined correctly

- Complicated parameter correlations can be implemented and tested

- Different types of data can be combined

- Parameter degeneracies can be broken

- Reduced number of degrees of freedom

\section{Disadvantages}

- Increased runtime of fits and uncertainty calculations

- Large memory is needed $\rightarrow$ multi-CPU calculations required

- Statistical weights of datasets have to be chosen

- Careful handling of fit-parameters required

TABLE 1. Advantages and disadvantages of fitting several datasets simultaneously.

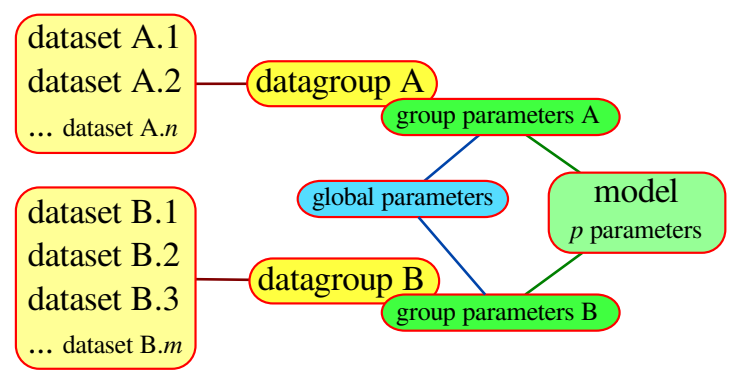

Figure 1. Terminology of simultaneous fits in ISIS. There are $n$ and $m$ simultaneous datasets, forming datagroup A and datagroup B, respectively. Each dataset has its own group parameters, resulting from a model with $p$ parameters. Some of the group parameters are the same for both datagroups and are called global parameters.

to, e.g., XSPEC 3, 4]. For example, user-defined fit-functions, as well as complex correlations between data and models, can be implemented. However, no functions are yet available for handling these correlations for a large number of parameters and datasets in an easy way.

Before we describe the technical realization of simultaneous data analysis in ISIS, we introduce new notations used by the implemented functions.

\subsection{Terminology}

The parameters of a model which is fitted to data either act on all datasets loaded into ISIS, or on an individual dataset. By defining parameters for each dataset and tying them to each other, parameters can be linked to multiple datasets similar to the approach chosen, e.g., in XSPEC.

Multiple datasets that are to be fitted with the same set of parameters are called a datagroup. The corresponding parameters are called group parameters. Global parameters denote parameters which act on all datagroups.
Figure 1 illustrates these definitions. In this example, a dataset requires a model with $p$ parameters. Simultaneous data, which can be described by the same parameters are available from $n$ detectors. These datasets define datagroup A with $p$ free parameters. Another group of data was recorded by $m$ detectors. These datasets define an individual datagroup B with, again, $p$ free parameters. During the analysis of the two groups, however, it turns out that a specific parameter seems to be equal for both data groups within the uncertainties. As a result, the two individual values for this parameter are tied to each other, resulting in a global parameter. This reduces the number of free parameters by one and the remaining group parameters can be constrained better.

\subsection{DATA- AND ANALYSIS FUNCTIONS}

Since simultaneous fits can have large numbers of fit parameters connected by a complicated logic, we provide a collection of all functions necessary to initialize and perform simultaneous fits in ISIS2 A simultaneous fit is initialized via

simfit $=$ simultaneous_fit ();

where simultaneous_fit returns a structure (Struct_Type), which has to be assigned to a variable, here simfit. The structure contains several functions and fields to handle simultaneous fits. The documentation of each function is available using the help-qualifier. Some important functions are described in the following.

simfit.add_data(filenames);

This defines a datagroup and loads the spectra given by filenames, which must be an array of strings. The function also allows other data than spectra to be loaded or defined.

simfit.fit_fun(model);

The string model defines the fit-function to be used for all datasets. Here, the placeholder \% can be used instead of a component instance. In this case individual group parameters are applied automatically to each datagroup.

simfit.set_par_fun(parameter, function);

This is probably one of the most useful functions. Like the ISIS intrinsic function, the value of the parameter is determined by the given function. The \%-placeholder can be used within the string parameter to apply the function to the corresponding parameter of each data group. However, the function may also contain other parameters or even a single parameter name. In the latter case, if the function is

\footnotetext{
${ }^{2}$ These functions are available as part of the ISISscripts, a collection of useful functions, which can be downloaded at http://www.sternwarte.uni-erlangen.de/isis [2015-05-06].
} 
also applied to all datagroups (using the \%), the single parameter is treated as global parameter from now on.

Because a simultaneous fit results in a large number of parameters, a single call to a fit-routine (fit_counts) will take a long time. In the example from the previous section, the final model fitted to the data consists of $(n+m) \times p$ parameters, where only $2 p-1$ are free. To reduce the runtime of a fit, two fit-routines are implemented within the simultaneousfit-structure.

\section{simfit.fit_groups (groupID);}

Instead of perfoming a $\chi^{2}$-minimization of all parameters and datasets, this function loops over all datagroups and fits only the associated parameters (group parameters). If a group is specified by the optional groupID, then only the group parameters of this particular group are fitted.

$$
\text { simfit.fit_global(); }
$$

Instead of fitting the group parameters, this function fits the global parameters only. Since all defined data groups have to be taken into account, the fit usually takes longer than fitting the group parameters.

\subsection{UNCERTAINTY CALCULATIONS}

As has already been mentioned, the runtime of simultaneous fits takes longer than fitting a single dataset only. Thus, uncertainty calculations of parameters, where the range of a certain parameter has to be found corresponding to a change in $\chi^{2}$, will be affected dramatically by the high runtime. Furthermore, it is necessary to distinguish between group parameters and global parameters. We recommend computing the uncertainty intervals for each parameter on a different machine, e.g., by using [5] or mpi_fit_pars and the SLmpi module ${ }^{3}$. We compared the runtime of a parallel uncertainty calculation in ISIS with a serial approach in XSPEC. Estimating the uncertainties of 10 parameters in parallel (i.e., on 10 cores) is faster by a factor of more than $3(21 \mathrm{ks}$ vs. $60 \mathrm{ks})$. Additionally, the calculations in ISIS resulted in a better $\chi_{\text {red }}^{2}$ because the parameter ranges being scanned are larger in the parallel approach.

Group parameters depend on a single datagroup only. As a consequence, all other datagroups and therefore all other group parameters can be ignored during the uncertainty calculation. Unfortunately, this not the case for global parameters. During the analysis of GRO J1008-57 (see Section 4), the uncertainties of the global parameters were calculated by revealing the $\chi^{2}$-landscape of each global parameter by individual fits. Subsequently, each landscape was interpolated to find the $\Delta \chi^{2}$-value of interest

http://www.sternwarte.uni-erlangen.de//git.public/ ?p=slmpi.git [2015-04-01] (e.g., $\Delta \chi^{2}=2.71$ corresponding to the $90 \%$-confidence level). In this way the runtime of an uncertainty calculation of a single global parameter could be reduced significantly. Note that depending on the model and on the amount of data, such a computation can take up to several days.

\section{Applications}

There are various applications of simultaneous fits and data analysis. Besides determining specific parameters that seem to be constant over time by all available data, more physical questions can be tackled. For example, if a physical property of the object of interest results in multiple observables:

- the geometry of the accretion column in accreting neutron star X-ray binaries affects the line shape of cyclotron resonant scattering features (CRSF) 6] and also the pulse profile shape (Falkner et al., in prep.).

Furthermore, instead of deriving physical properties from parameters after fits have been performed, these properties can be directly fitted to the data by implementing the dependency on the model parameters:

- the components in radio maps of jets in active galactic nuclei move at certain velocities. Assuming a constant velocity of the jet components, the velocity itself could be a global fit parameter [7].

- in the sub-critical accretion regime of neutron stars, the spectrum is believed to harden with increasing luminosity [8]. Any possible dependency between power-law shape and luminosity could be fitted simultaneously with multiple spectra.

\section{The Example GRO J1008-57}

As an example of a successful simultaneous fit we will briefly summarize the results of our analysis of GRO J1008-57 using almost all available X-ray spectra and -lightcurves. This transient high-mass X-ray binary consists of a neutron star orbiting a Be-type optical companion. For further details of the system and also for the results of the analysis see [9] and references therein.

Since sources are only visible for a small fraction of their full orbit, it is challenging to obtain the orbital parameters of transient X-ray binaries by analyzing, e.g., the pulse arrival times [10, 11]. Thus, an observed shift in the orbital phase with respect to the initial orbital parameters can be fitted with either a different orbital period or with a different time of periastron passage. This leads to a parameter degeneracy, which can be visualized by a contour map of the $\chi^{2}$-landscape of these parameters. The resulting contour map shows that both parameters are degenerated statistically (i.e., the ellipsoidal contour lines are tilted).

However, the outburst times of the source are clearly connected to the periastron passage. Performing a simultaneous fit of the pulse arrival times and the 


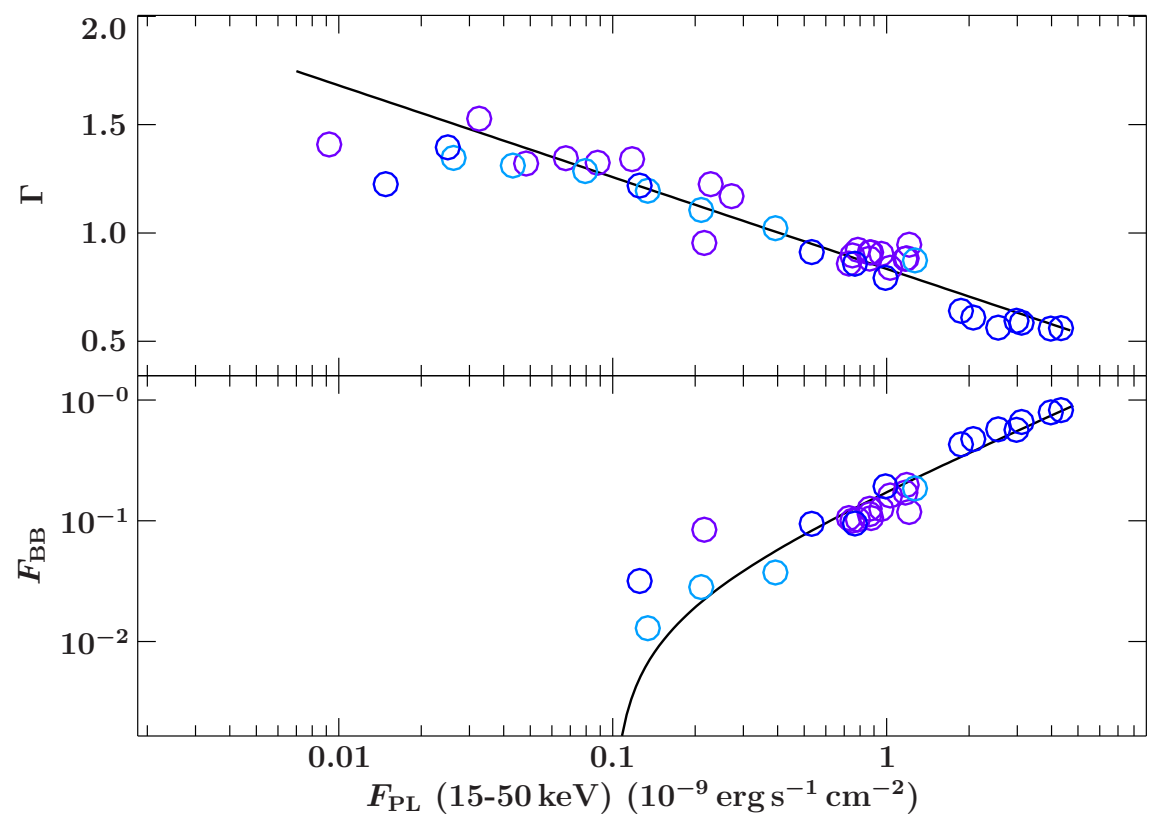

Figure 2. A fit (black lines) of the power-law index $\Gamma$ and black body flux $F_{\mathrm{BB}}$ as functions of power-law flux $F_{\mathrm{PL}}$. The different colors correspond to different outbursts.

outburst times reduces the parameter degeneracy and results in much better constrained parameters (by a factor of about 2-3), as is shown by recalculated contour map.

Initial fits of the spectra of three outbursts of GRO J1008-57 in 2005, 2007 and 2011 with an absorbed cutoff power-law and an additional black body component showed that the folding energy $E_{\text {fold }}$, as well as the black body temperature $k T$, are independent of time within uncertainties. In particular, it seems that they do not change between different outbursts, i.e., these parameters are constant properties of the source.

These parameters are therefore set as global parameters using simfit.set_par_fun and their values are determined by all available data. In addition, further parameters can be treated as the global parameters [see 9, for more details]. Finally, each observation is described by 3 group parameters only $(\approx$ degrees of freedom for each datagroup, the global parameters contribute marginally), which are the power-law flux $F_{\mathrm{PL}}$, the black body flux $F_{\mathrm{BB}}$, and the photon index $\Gamma$. The latter two parameters correlate strongly with $F_{\mathrm{PL}}$, but show no dependency on the outburst time or the outburst shape. This correlation can be fitted to describe the spectrum of GRO J1008-57 by only one parameter: the power-law flux $F_{\mathrm{PL}}$. The fit is shown in Fig. 2 and its values are given in Section 4.2 of [9].

As has already been mentioned in Section 2.3 the runtime for the uncertainty calculations of the global parameters is increased dramatically. In the case of this analysis, the $\chi^{2}$-landscape produced by taking all 68 spectra into account was interpolated to estimate the uncertainties. The calculation of a single global parameter took $\sim 7$ days on 100 CPUs (16320 CPUh).

\section{OUTLOOK}

Although simultaneous fits have already been applied successfully to real data (see Section 4 and [9]), the routines and functions are still under development. We recommend to pull the isisscripts-GIT-repository ${ }^{2}$ regularly to be up-to-date.

There are, however, some caveats according to Table 1 that one should be aware of (as with any routine, not just our ISIS implementation). In particular, the runtime still has to be reduced. One way to achieve this is by performing the fit on multiple CPUs, e.g., one CPU handles one dataset or datagroup. This has not been implemented yet because the dependencies of the datasets on each other require data exchange between the processes on the different machines. Additionally, the question of weighting the data is currently under discussion. The weight depends on the number of datapoints available in each dataset (or -group) as well as their uncertainties - but what does this mean for its importance, i.e., for its effect on the model parameters? These remaining issues have to be clarified and the respective solutions will be published in the future.

\section{ACKNOWLEDGEMENTS}

M. Kühnel was supported by the Bundesministerium für Wirtschaft und Technologie under Deutsches Zentrum für Luft- und Raumfahrt grant 50OR1113. We thank John E. Davis for developing the SLXfig package, which was used to create all figures shown in this paper.

\section{REFERENCES}

[1] J. C. Houck, et al. ISIS: An interactive spectral interpretation system for high resolution X-ray spectroscopy. In N. Manset, et al. (eds.), Astronomical 
Data Analysis Software and Systems IX, vol. 216 of Astron. Soc. of the Pacific Conf. Series, p. 591. 2000.

[2] M. S. Noble, et al. Beyond xspec: Toward highly configurable astrophysical analysis. Publ of the Astron Soc of the Pacific 120:821-837, 2008.

[3] R. A. Schafer. XSPEC, an X-ray spectral fitting package : version 2 of the user's guide. European Space Agency, 1991.

[4] K. A. Arnaud. Xspec: The first ten years. In G. H. Jacoby, et al. (eds.), Astronomical Data Analysis Software and Systems V, vol. 101 of Astron. Soc. of the Pacific Conf. Series, p. 17. 1996.

[5] M. S. Noble, et al. Using the parallel virtual machine for everyday analysis. In C. Gabriel, et al. (eds.), Astronomical Data Analysis Software and Systems XV, vol. 351 of Astron. Soc. of the Pacific Conf. Series, p. 481. 2006.
[6] G. Schönherr, et al. A model for cyclotron resonance scattering features. A\&SA 472:353-365, 2007.

[7] C. Grossberger, et al. $A \mathscr{E} A$ In prep.

[8] P. A. Becker, et al. Spectral formation in accreting X-ray pulsars: bimodal variation of the cyclotron energy with luminosity. $A \& A$ 544:A123, 2012.

[9] M. Kühnel, et al. GRO J1008-57: an (almost) predictable transient X-ray binary. A $\mathscr{G} A$ 555:A95, 2013.

[10] J. E. Deeter, et al. Pulse-timing observations of Hercules X-1. ApJ 247:1003-1012, 1981.

[11] P. E. Boynton, et al. Vela X-1 pulse timing. I determination of the neutron star orbit. ApJ 307:545-563, 1986. 